\title{
La Décentralisation comme Ambition Multiple
}

Nassirou Bako-Arifari et Pierre-Joseph Laurent

\section{(2) OpenEdition}

\section{Journals}

Édition électronique

URL : http://journals.openedition.org/apad/552

DOI : 10.4000/apad.552

ISSN : 1950-6929

Éditeur

LIT Verlag

Édition imprimée

Date de publication : 1 mai 1998

Référence électronique

Nassirou Bako-Arifari et Pierre-Joseph Laurent, «La Décentralisation comme Ambition Multiple », Bulletin de l'APAD [En ligne], 15 | 1998, mis en ligne le 19 décembre 2006, consulté le 08 septembre 2020. URL : http://journals.openedition.org/apad/552 ; DOI : https://doi.org/10.4000/apad.552

Ce document a été généré automatiquement le 8 septembre 2020

Bulletin de l'APAD 


\title{
La Décentralisation comme Ambition Multiple
}

\author{
Nassirou Bako-Arifari et Pierre-Joseph Laurent
}

1 Consacrer ce bulletin au thème de la décentralisation répond à plusieurs préoccupations ${ }^{1}$. D'abord, il s'agit de rendre compte du colloque organisé par le réseau APAD "Décentralisation et pouvoirs locaux" en collaboration avec l'Université Catholique de Louvain-La-Neuve (Unité d'anthropologie de l'Institut d'Etudes du Développement) et qui s'est déroulé du 20 au 21 novembre 1997 à Louvain-la-Neuve en Belgique sur le thème "Les dimensions sociales et économiques du développement local et la décentralisation en Afrique au sud du Sahara". Cette rencontre s'inscrivait aussi dans le cadre des préparatifs du grand rendez-vous apadien de Cotonou (Bénin), les Ve journées APAD sur le thème "Décentralisation, pouvoirs locaux et réseaux sociaux" du 26 au 28 novembre $1998^{2}$. Les contributions présentées ici sont pour l'essentielle fruit de cette rencontre de Louvain.

2 Ensuite, il s'agit de poursuivre, dans la ligne du Bulletin de l'APAD 14 consacré à la décentralisation au Mali (Kassibo 1998) mais en diversifiant les pays et les perspectives, la réflexion sur cette problématique dont l'actualité ${ }^{3}$ concerne à la fois la science et le développement et qui semble devenir dominante dans cette fin de la décennie 1990, particulièrement en Afrique. Ce ne sont pas seulement des cas africains (même s'ils sont majoritairement issus des pays francophones d'Afrique de l'Ouest : Bénin, Burkina Faso, Niger, Sénégal) qui sont présentés ici mais aussi un cas antillais (Dorner sur la décentralisation en Haïti) et les contributions englobent aussi bien le milieu rural que le milieu urbain (Tall sur les délégués de quartiers à Dakar au Sénégal), une ouverture macro-politique proposant une économie politique de la décentralisation dans le contexte de la globalisation (Laurent \& Peemans) et deux contributions centrées sur des contextes de pré-décentralisation: l'une relative au fonctionnement d'une arène politique villageoise au Bénin marqué par des innovations institutionnelles importantes dans une perspective de "dé-centralisation" autonome devançant ainsi la politique officielle en la matière (Le Meur) et l'autre sur projets de développement et chefferie au Niger (Olivier de Sardan), contributions dont les conclusions informent sur 
la nature des arènes locales sur lesquelles vont intervenir les politiques de décentralisation en cours d'élaboration.

Il s'agit enfin de positionner cette problématique dans le débat général dans les sciences sociales, notamment dans le champ de la socio-anthropologie politique et du développement.

Le contexte macro-sociologique de la décentralisation

4 La vague de contestation populaire ayant conduit les acteurs du "politique par le bas" parfois sans leadership clairement identifié, à l'assaut des citadelles des régimes réputés dictatoriaux et de partis uniques à la fin des années 1980 et au début des années 1990 , conjointement avec les exigences issues du nouvel ordre international de la fin de la guerre froide et celles des politiques d'ajustement structurel, ont ouvert la voie à des politiques de réforme à la fois économique et politique des Etats africains dans le sens d'une plus grande orientation vers les impératifs du libéralisme politique et du marché, mais aussi vers une plus grande promotion de la participation politique des populations locales.

5 Ces événements macro-politiques ont débouché sur des changements de centres d'intérêts aussi bien du côté des bailleurs de fonds que de la communauté scientifique par rapport à la problématique générale du développement. Le modèle du "developmental state" et de la stratégie de développement monocentrique descendant (lop-down) ont montré leurs limites et désormais, c'est la participation des populations qui est devenue le point focal aussi bien pour les bailleurs de fonds que pour les acteurs de la coopération décentralisée (ONG et autres collectivités locales du Nord surtout). Dès lors, le discours des bailleurs de fonds et des institutions de "brainstorming" internationales s'est orienté vers une apologie tous azimuts des vertus de la démocratie mais aussi de la régulation par le marché (cf. The Carter Center 1989, Banque Mondiale 1989). Les nouvelles orientations ont même parfois pris l'allure de nouveaux slogans et de nouvelles conditionnalités du développement (et aussi de l'aide au développement) : désengagement de l'Etat, promotion de la société civile, promotion de la régulation par le marché, appui aux processus de démocratisation, lutte contre la corruption et exigence $d^{\prime \prime}$ 'accountability" et de transparence dans la gestion des affaires publiques à tous les niveaux. Bref, il s'agit d'établir des bases durables pour la "bonne gouvernance" (good governance; cf. World Bank 1992, Hyden \& Bratton 1992), nouveau concept fédérateur de tous toutes ces conditionnalités du développement et de l'aide au développement.

6 Si les processus de démocratisation - entendez des élections libres, transparentes et pluralistes - permettent de résoudre la question de la dévolution du pouvoir au sommet de l'Etat, l'opérationalisation dans les secteurs infra-étatiques de tous les autres paramètres de la bonne gouvernance ne peut se faire que par un transfert nécessaire sinon obligatoire de compétences des centres étatiques vers des collectivités territoriales autonomes (du moins dans l'esprit de ses promoteurs), afin d'assurer la participation politique des acteurs sociaux et politiques aux différentes échelles locales. C'est dans cette nouvelle orientation globale que la décentralisation est apparue comme un dispositif-cadre politico-institutionnel d'agencement de l'ensemble de ces paramètres du développement et de la participation politique. Les stratégies de développement participatif et de développement local acquièrent dans cette perspective une dimension nouvelle et plus large et les politiques de décentralisation comportent désormais une orientation économique et sociale très affirmée. Cependant, 
partisans de la décentralisation et défenseurs des stratégies éprouvées du développement local ne s'accordent pas sur les finalités de la décentralisation et leur affrontement a tout l'air d'un jeu à somme nulle. Le texte de Jacob dans ce Bulletin expose magistralement l'affrontement des arguments intellectuels et techniques de ces deux catégories d'acteurs et la façon dont les différents gouvernements (au Burkina et au Mali par exemple) en tirent parti pour ajuster à chaque fois les objectifs de la décentralisation à ceux de leur survie politique.

7 Peu importe que les acteurs engagés ou à engager dans ces politiques, et ceci du sommet à la base, aient des perceptions et des attentes différentes par rapport aux politiques de décentralisation et de développement local, l'essentiel à l'étape actuelle est, semble-t-il, de parvenir au compromis, disons au "consensus" sur la décentralisation (nouvelle version du "Washington consensus" dont parle Williamson 1993), sachant bien entendu qu'il n'y a de consensus que comme expression de la volonté de celui ou de ceux occupant les principales positions dans un champ de forces donné.

8 Dans ce contexte marqué par la "tendance dominante de l'idéologie de la globalisation" (Laurent \& Peemans), où partout l'économie post-fordiste se manifeste par des délocalisations d'entreprises, un maillage serré du capital international à travers les "mariages" de plus en plus fréquents de multinationales dans des domaines différents; et où l'Etat occidental devenu lui-même post-fordiste renonce à des pans assez importants de sa souveraineté, la décentralisation apparaît comme une sorte de conjugaison à échelle plus réduite de ces dynamiques macro-économiques et politiques. La stratégie de délocalisation devient une stratégie de décentralisation économique et de multiplication des lieux de production comme la décentralisation politique elle aussi agit par le renforcement des collectivités locales. Le local finalement (ou paradoxalement) apparaît comme un dénominateur commun (Laurent \& Peemans) à la globalisation et à la décentralisation. Un autre dénominateur commun est la renonciation (toutes proportions gardées) à des attributs considérés jusque-là comme relevant de la souveraineté des Etats. Dans les Etats occidentaux, c'est l'abdication de la souveraineté sur les frontières nationales devant la mondialisation financière et commerciale, tandis que à l'intérieur des Etats africains, en plus de cette dimension, il y aussi l'obligation de transfert de compétences des administrations étatiques centrales vers les collectivités locales, créant ainsi des "para-souverainetés" locales pour utiliser le terme de von Trotha.

9 Le colloque de Louvain est donc intervenu dans ce contexte et se voulait un cadre de réflexion et d'examen de toutes ces considérations-équations souvent normatives qui sont associées dans des partitions diverses aux thématiques du développement et de la décentralisation, par leur confrontation avec le quotidien des populations "cibles" (pour utiliser une rhétorique de "projet") et des politiques étatiques de décentralisation (élaboration, application, expériences capitalisées) dans les arènes aussi bien locales que nationales. C'est à ce pari que s'est attelée la centaine de participants au colloque, qui s'est déroulé exclusivement en plénière comme pour créer une osmose complète entre toutes les personnes présentes et pour offrir l'opportunité à chacun d'écouter intégralement l'autre et de participer pleinement aux débats sans la médiation récapitulative des responsables d'atelier.

La décentralisation comme objet d'investigation des sciences sociales 
10 La légitimité de la décentralisation comme objet d'investigation socio-anthropologique pour l'APAD découle d'une série de coïncidences de préoccupations entre chercheurs en sciences sociales et praticiens du développement, les deux piliers fondateurs de l'association et qui balisent depuis 1991 son orientation et ses actions. La décentralisation s'inscrit dans le cadre de thématiques-carrefours entre ces deux groupes d'acteurs qui s'intéressent aux sociétés africaines. ${ }^{4}$

- La décentralisation est indissociable la problématique des pouvoirs locaux et de toute la problématique classique de l'anthropologie politique, à savoir celle de l'Etat, entendu non plus en termes d'existence ou d'absence de cette institution, mais plutôt en termes de réforme de l'Etat. ${ }^{5}$ Le "trop d'Etat" est devenu aujourd'hui la "bête noire" des concepteurs des politiques de développement et tout incite désormais dans l'environnement international et à l'intérieur des Etats au "moins d'Etat" ou disons à "l'Etat minimal fonctionnel" (cf. Laurent \& Peemans). Le "moins d'Etat, mieux d'Etat" crée indirectement mais sûrement un nouveau type d'Etat, "l'Etat local" avec ses enjeux, ses acteurs et ses contours propres, qui pourrait devenir de plus en plus autonome, au moins pour ce qui est du domaine de la compétition politique (cf. Blundo). Cet Etat local "redistribue" à sa façon du centre vers les échelles infra-étatiques la question de la séparation et des relations entre les sphères du "public" et du "privé", qui est restée l'une des questions non résolues par l'Etat africain (ou disons les Etats africains). Le texte de Jacob revient largement sur ce débat et ses enjeux dans les politiques de décentralisation.

- La décentralisation pose également la problématique des recompositions sociales et politiques des arènes à la fois nationales et locales de pouvoir et appelle à l'analyse des processus sociaux et des dynamiques politiques locales qu'elle pourrait déclencher dans les contextes d'élaboration des politiques de décentralisation, et de ceux en cours dans les contextes de décentralisation avancés. Elle offre alors une opportunité de repositionnement aux différents acteurs sociaux, économiques et politiques locaux à travers des dynamiques tantôt clientélistes et factionnalistes de construction de réseaux sociaux divers (les contributions de Blundo et de Le Meur explicitent cette "la participation factionnelle"), tantôt de confrontation ou de négociation dans lesquelles certaines institutions locales non prises en compte par les instances décentralisées peuvent se révéler plus pertinentes comme échelon de résolution de conflit et donc dominantes par rapport aux institutions officielles (cf. la contribution de Laurent aux journées de Louvain-La-Neuve, et Laurent 1996), dont les "légitimités municipales et de mobilisation" (Olivier de Sardan) sont encore en train de se faire. Que les chefs traditionnels soient plus prépondérants dans les affaires foncières que les Communautés rurales sénégalaises investies officiellement depuis un quart de siècle de la compétence en la matière n'est qu'une des manifestations parmi tant d'autres de ces pratiques de cette négociation locale.

11 Par ailleurs, la promotion de la bonne gouvernance par la décentralisation peut apparaître comme un simple slogan, d'abord parce que les instances décentralisées ne bénéficient pas du jour au lendemain d'une légitimité fiscale suffisante pouvant leur permettre d'opérer les ponctions fiscales nécessaires pour assurer la viabilité des collectivités locales, mais aussi et surtout parce que le fonctionnement même des institutions décentralisées au quotidien semble être porteur et producteur d'opportunités de détournement, de corruption et de pratiques diverses de malversations (octroi complaisant de marchés publics, détournement de projets par les élus locaux, création d'une nouvelle élite ou de privilégiés locaux qui se légitiment par leur appartenance aux instances décentralisées, bref autant d'effets induits qui finissent par mettre les pratiques quotidiennes des instances décentralisées à des 
années-lumière des objectifs et des attentes initialement formulés). En tout cas, la contribution de Blundo, qui n'est pas spéculative mais fondée sur une étude empirique est très explicite quant à ces mécanismes et ces pratiques locales dans les communautés rurales sénégalaises.

- La décentralisation est rarement perçue en soi comme problématique se suffisant à elle-même. Bien au contraire, elle est mise chaque fois en relation avec des problématiques connexes : décentralisation et développement local et/ou participatif, décentralisation et coopération décentralisée au développement, décentralisation et pouvoirs locaux, décentralisation et démocratie locale, décentralisation et bonne gouvernance, etc. Bref autant de partitions qui conduisent parfois certains à vite faire le pas vers des corrélations mathématiques transformant la décentralisation en un "dispositif" à tout faire et malléable en tous sens et comme une solution miracle aux nombreux défis du développement et à la faillite du modèle du developmental state et de la gestion des affaires publiques locales. Les contributions présentées dans ce volume incitent plutôt à tempérer les ardeurs et à percevoir la décentralisation comme un outil pédagogique de mise en oeuvre de politiques ou d'interventions de développement ainsi que comme une problématique fédératrice de nombreuses préoccupations scientifiques et de développement. La décentralisation ou les décentralisations doi(ven)t être perçue(s) en contexte et non pour elle(s)-même(s).

- La décentralisation est également vue comme moyen de renouvellement des problématiques du "local-level politics", du "local-central politics", ou encore des relations "centre-periphérie" et de l"'Etat-paysan", etc. ${ }^{6}$ qui dans les années 1960, 1970 et même 1980 ont dominé les recherches en anthropologie politique et économique sur les sociétés des pays en développement, africaines en particulier. Désormais, ce ne sera plus l'analyse du degré d'autonomie du local par rapport au central qui doit préoccuper mais plutôt celle touchant à la manière dont l'autonomie "octroyée" au local est insérée dans les dynamiques politiques, sociales et économiques locales. Il s'agit là, comme en douce, d'une révolution paradigmatique qui demande toutefois encore du temps pour être appréciée à sa juste valeur. Les résistances des pouvoirs centraux par rapport aux politiques de décentralisation dans un certain nombre de pays tout comme la volonté de limiter le nombre d'échelons de collectivités décentralisées, ou encore les hésitations à définir clairement ou à transférer beaucoup de compétences aux collectivités décentralisées ou à y associer toutes les institutions locales à ce processus comme le souhaitent les acteurs locaux eux-mêmes sont autant d'aspects qui ont préoccupé certains contributeurs dans ce volume. Les textes de Pierre-Joseph Laurent $(1996,1997)$ et les analyses de Jacob à propos du Burkina Faso montrent combien il n'est pas aisé de concilier les attentes exprimées par les acteurs locaux par rapport à la décentralisation, notamment en milieu rural, les propositions des experts et les objectifs des pouvoirs publics qui ne sont pas toujours clairement définis mais plutôt englués dans des discours laissant des issues de secours entrouvertes à ceux qui contrôlent l'appareil du pouvoir d'Etat. Et lorsque certaines compétences sont officiellement transférées par lois ou décrets, elles sont replacées indirectement sous contrôle par des mécanismes bureaucratiques et juridiques complexes (cf. le cas du Sénégal avec Blundo). Tout cela constitue autant d'interrogations que les contributions présentées ici ont essayé d'aborder.

- La décentralisation comme une thématique de l'institution-building et de l'empowerment apparaît, quoiqu'en creux, dans les différentes contributions et toutes l'évoquent indirectement. La création des collectivités décentralisées est à plus d'un égard une innovation institutionnelle importante dans nombre de milieux, où elles interviennent. La question du choix des institutions pertinentes (Laurent 1997, pour le milieu rural 
burkinabè), leur fonctionnement quotidien (Blundo sur le Sénégal), leur caractère formel ou informel (les délégués de quartier de Dakar étudiés par Tall), introduisent tous à cette problématique ; et montrent que les stratégies de "circuits courts" qui sous-tendent souvent les interventions de développement sous-estiment la réalité du pluralisme institutionnel des sociétés africaines et que la décentralisation ne se fera que dans ce contexte et en comptant avec lui (voir contribution de Jacob sur ce point).

- Enfin, la décentralisation apparaît comme une thématique de relation interculturelle, en ce sens que les débats sur les politiques de décentralisation tournent autour de modèles déjà éprouvés dans des contextes socio-historiques totalement différents de ceux des différents pays africains contemporains : expériences américaine, allemande et française (sans compter le modèle du local government britannique en Afrique anglophone). Encore une fois, tout se passe comme si l'on occultait le fait que les différenciations entre sphères "publique" et "privée" et les traditions de démocratie représentative à l'échelle locale sont toutes culturellement indexées : la volonté actuelle de les "imposer" (décentralisation sous contrainte) semble faire l'impasse sur les sources réelles de la légitimité du pouvoir dans les espaces infra-étatiques. Le combat réel sera alors celui de la validation locale des "légitimités" électorales locales au yeux de la majorité. Quels types de capital social et quels facilitateurs culturels paraissent finalement être déterminants pour légitimer des "positions électorales" et pour exercer les fonctions qui leur sont liées ? La décentralisation permet de réexaminer ces dimensions sociales et culturelles du pouvoir, notamment dans les sociétés rurales africaines.

Tout ceci fait plutôt de la décentralisation une vaste problématique fédératrice de sous-problématiques diverses et une occasion de réexamen ou de réévaluation de perspectives anciennement développées sur l'Etat et sa relation avec la société en général, sur le pouvoir et le développement local. Et c'est là que se situe la richesse de cet objet et son caractère intellectuellement stimulant, mais aussi sa dimension pratique, nouveau défi pour les praticiens du développement. Tout incite à ne pas faire de la décentralisation une nouvelle boîte noire à l'instar la thématique du "développement". Par sa richesse et la pluralité de perspectives qu'elle autorise, elle devrait échapper à toute tentative de récupération par quelque théorie mono-directionnelle dure que ce soit (et l'extinction du conflit des paradigmes scientifiques va plutôt dans ce sens).

\section{BIBLIOGRAPHIE}

Alternatives Sud vol. IV (3) 1997. "Pouvoirs locaux et décentralisation". Louvain-La-Neuve - Paris : Centre Tricontinental-L'Harmattan.

Balandier, G. 1969. Anthropologie politique. Paris : Presses Universitaires de France.

Banque Mondiale 1989. L'Afrique sub-saharienne: de la crise à une croissance durable : étude de prospective à long terme. Washington : Thé World Bank. 
Barker, J. \& P. Carstens (eds.) 1970. "Local-Central Politics". Canadian Journal of African Studies 1 (4).

Bierschenk, T. \& J.-P. Olivier de Sardan (dir.) 1998. Les pouvoirs aux villages. Le Bénin rural entre démocratisation et décentralisation. Paris : Karthala (sous presse).

Binsbergen, W. van, Reyntjens \& Hesseling (eds.) 1986. State and local community in Africa. Les cahiers du CEDAF (2-3-4), Bruxelles : CEDAF. Easton, D. 1959. "Political Anthropology". Annual Review of Anthropology 1. Geschiere, P. 1982. Village Communities and the Slate, Changing relations among the Maka of Southeastern Cameroon since the Colonial Conquest. London : Kegan.

Hyden, G \& Bratton, M. (eds.) 1992. Governance and Politics in Africa. Boulder : Lynne Riener.

Jacob, J.-P. \& G. Blundo, en collaboration avec N. Bako-Arifari, K. Borhaug et P-J Laurent, Socio-anthropologie de la décentralisation en milieu rural africain. Bibliographie sélective et commentée, Genève, IUED (Itinéraires, série "Notes et Travaux" 49).

Kassibo, B. (ed.) 1998. "La décentralisation au Mali : état des lieux". Bulletin de l'APAD 14 (dossier thématique), Hamburg, Lit Verlag.

Laurent, P.-J. 1996. "Développement local, stabilité politique et décentralisation. l'exemple du Burkina Faso". Bulletin de l'APAD 12 : 158-161.

Laurent, P.-J. 1997. L'état de la décentralisation au Burkina Faso : soutien du développement local et opportunité de re-légitimation de l'Etat. Contribution au séminaire "Les dimensions sociales et économiques du développement local et la décentralisation en Afrique au Sud du Sahara", Louvain-La-Neuve (20-22 novembre 1997), APAD-Université Catholique de Louvain-La-Neuve.

Le Meur, P.-Y 1998. "Compte-rendu du séminaire «Les dimensions sociales et économiques du développement local et la décentralisation en Afrique au Sud du Sahara»". Bulletin de l'APAD : 139-143.

Lewellen, T.C. 1983. Political Anthropology : An Introduction. South Hadley : Bergin \& Garvey. Swartz, M. (ed.) 1968. Local-level politics. Chicago : Adline.

Swartz, M., V. Turner \& A. Tuden (eds.) 1966. Political Anthropology. Chicago : Adline.

The Carter Center 1989. Beyond Autocracy in Africa. Seminar on the African Governance Programme, Atlanta : Emory University.

Williamson, J. 1993. "Democracy and the «Washington Consensus»". World Development 21 (8) : 1329-1336.

World Bank 1992. Governance and Development. Washington : The World Bank.

\section{NOTES}

1.Ce dossier thématique a été réalisé en étroite collaboration entre les éditeurs et un secrétariat de rédaction composé de Jean-Pierre Jacob, Giorgio Blundo et Laurent Monnier de l'IUED de Genève.

2.De fait, un certain nombre de contributions étaient des textes provisoires que les auteurs retravailleront à la lumière des commentaires faits par les uns et les autres et qui seront présentés à Cotonou. Ceci fait partie de la stratégiepédagogique de l'APAD à l'égard de ses membres. Chaque colloque APAD est précédé d'un pré-colloque préparatoire, mais qui cette fois-ci à Louvain a pris un caractère encore plus important 
par le nombre des participants (cf. le compte-rendu de P.-Y Le Meur dans le Bulletin de l'APAD 14 ; Le Meur 1998). Le Bulletin de l'APAD 16 (sous la direction de Giorgio Blundo et Roch Mongbo), qui paraîtra à la veille des journées de Cotonou et présentera comme à l'accoutumée les textes qui seront présentés en plénière, prendra aussi en compte certaines des contributions de Louvain.

3.Pour une revue récente de la littérature sur la décentralisation centrée sur l'Afrique au Sud du Sahara, cf. Jacob, Blundo et al 1997, Alternatives Sud 1997. Par ailleurs, de nombreux séminaires sont organisés sur la décentralisation en Afrique (séminaires nationaux, régionaux, etc.) par les bailleurs de fonds comme la Fondation Konrad Adenauer qui en a fait un point de concentration de son aide en Afrique Occidentale et Centrale ou encore de la Coopération Suisse (DDC). Des institutions régionales sont créées pour s'occuper exclusivement de cet objet comme le Programme de développement municipal (PDM) basé au Bénin. Pour une étude récente sur ce thème en relation avec les processus de démocratisation, cf. Bierschenk \& Olivier de Sardan (dir.) 1998.

4.Certains parmi les intervenants du séminaire de Louvain ont joué ou jouent encore des rôles de conseillers auprès de certains gouvernements africains dans l'élaboration de leurs politiques de décentralisation, d'autres acteurs de la coopération au développement y étaient en qualité de observateurs actifs (posant des questions et participant aux débats).

5.Sur ce point cf. Balandier 1969. Easton 1959. Lewellen 1983.

6.Sur ces thématiques, cf. Swartz (ed.) 1968, Swartz et al. (eds.) 1966. Barker \& Carstens (eds) 1970, Binsbergen et al. (eds) 1986, Geschiere 1982.

\section{AUTEURS}

\section{NASSIROU BAKO-ARIFARI}

Johannes Gutenberg-Universitîit, Institut fur Ethnologie und Afrikastudien, Forum 6, D-55099 Mainz (Allemagne). Tél. +49 (06131) 39 4580. Fax. +49 (06131) 393730 bakoarif@mail.uni-mainz.de

\section{PIERRE-JOSEPH LAURENT}

Université Catholique de Louvain, Place Montesquieu 3, B-1348 Louvain-La-Neuve (Belgique). Tél. +32 (64) 6774 39. Fax. +32 (10) 472805 - laurent@dvlp.ucl.ac.be 